\title{
Modeling and Availability Analysis of Cement Manufacturing Plant Subject to Coverage Factor and Human Failure
}

\author{
Reena \\ Research Scholar, Department of Mathematics, \\ Shri J.J.T. University, JhunJhunu, Rajasthan \\ Assistant Professor, Government College Bahu, \\ Jhajjar, Haryana, India
}

\author{
Vineeta Basotia \\ Assistant Professor, Department of Mathematics, \\ Shri J.J.T. University, JhunJhunu, Rajasthan
}

\begin{abstract}
The main aim of the present study is to perform availability analysis of a cement manufacturing plant using concepts of coverage factor and human failure. For this purpose, a mathematical model has been developed using Markovian birth-death process. In cement manufacturing plant various subsystems arranged in series structure. Sufficient repair facility always available with plant. All time dependent random variables are statistically independent and exponentially distributed. The repairs are perfect. The differential-difference equation has been solved using R-K method of fourth order. Numerical and graphical results have been obtained to highlight the importance of the study.
\end{abstract}

\section{Keywords}

Cement Manufacturing Plant, Coverage Factor, Availability, Mathematical Model.

\section{INTRODUCTION}

Cement is a vital development material in industrialization and human civic establishments. It sets, solidifies, and ties different materials and blended in with sand and items concrete. Low quality of framework can cause in building breakdown, street mishaps and other destructive occurrences. So, with the proper planning and management great nature of materials is required. Nature of any material can be improved by examining its availability and performance. A lot of researchers have examined the reliability and availability in the manner to improve the performance of their models. Saini and Kumar (2019) analysed performance of evaporation system in sugar industry using RAMD analysis. Kumar et al. (2019) analyzed rreliability, maintainability and sensitivity analysis of physical processing unit of sewage treatment plant. Kumar and Saini (2018) performed fuzzy availability evaluation of a marine power plant. Dahiya et al. (2019) developed a mathematical modeling and performance evaluation of A-Pan crystallization system in a sugar industry. Gupta et al. (2020) suggested stochastic model for operational availability analysis of generators in steam turbine power plants. Goyal, et al. (2020) carried out rreliability, maintainability and sensitivity analysis of biological and chemical processing unit of sewage treatment plant. Saini et al. (2020) proposed a study of microprocessor systems using RAMD approach. Saini and Kumar (2020) developed a stochastic model of a single-unit system operating under different environmental conditions subject to inspection and degradation. Dahiya et al. (2019a, 2019b) analysed processed industries like feeding system and harvesting system. Garg et al. (2020) developed stochastic model for a two non-identical units' redundant system with preventive maintenance and priority. Barak et al. (2017) studied a cold standby system with conditional failure of server.

By keeping all facts in mind, here an effort has been made to analyze the availability of a cement manufacturing plant under the concepts of coverage factor and human failure. The concept of coverage factor plays an important role in performance and availability of systems. The human failure also influences the system's performance. It is assumed that in the beginning of the system the human failure can occurs. Suitable redundancy concepts have been utilized as and when required and standby unit reduced the capacity of the plant. It is assumed that sufficient repair facility always available with system to do repair, maintenance of the plant and treatment of human failure. All the failure and repair rates follow exponential distribution. The Markovian birth-death process has been utilized to develop a mathematical model for the cement manufacturing plant and Chapman-Kolmogorov differential difference equation have been derived. The numerical results for availability and profit function have been derived for a particular set for parameters. The derived results can be proved helpful for system designers and management personals.

\section{STATE TRANSITION DIAGRAM AND PATH PARAMETER}

The failure and repair rates between states are considered as follows:

Table 1: Failure and repair rates

\begin{tabular}{|l|l|l|l|l|}
\hline S.No. & State $i$ & State $j$ & Path Parameter $i$ to $j$ & Path Parameter $j$ to $i$ \\
\hline 1 & $A B C D E F$ & $A B C D_{1} E F$ & $\alpha_{4} \gamma$ & $\mu_{4}$ \\
\hline 2 & $A B C D_{1} E F$ & $A B C D E F$ & $\mu_{4}$ & $\alpha_{4} \gamma$ \\
\hline
\end{tabular}




\begin{tabular}{|l|l|l|l|l|}
\hline 3 & $A B C D E F$ & $a B C D E F$ & $\alpha_{1}(1-\gamma)$ & $\mu_{1}$ \\
\hline 4 & $A B C D E F$ & $A b C D E F$ & $\alpha_{2}(1-\gamma)$ & $\mu_{2}$ \\
\hline 5 & $A B C D E F$ & $A B c D E F$ & $\alpha_{3}(1-\gamma)$ & $\mu_{3}$ \\
\hline 6 & $A B C D E F$ & $A B C D e F$ & $\alpha_{5}(1-\gamma)$ & $\mu_{5}$ \\
\hline 7 & $A B C D E F$ & $A B C D E f$ & $\alpha_{6}(1-\gamma)$ & $\mu_{6}$ \\
\hline 8 & $A B C D E F$ & Human Failure & $\alpha_{H}(1-\gamma)$ & $\mu_{H}$ \\
\hline 10 & $A B C D_{1} E F$ & $A b C D_{1} E F$ & $\alpha_{2}(1-\gamma)$ & $\mu_{2}$ \\
\hline 11 & $A B C D_{1} E F$ & $a B C D_{1} E F$ & $\alpha_{1}(1-\gamma)$ & $\mu_{1}$ \\
\hline 12 & $A B C D_{1} E F$ & $A B C D_{1} e F$ & $\alpha_{5}(1-\gamma)$ & $\mu_{5}$ \\
\hline 13 & $A B C D_{1} E F$ & $A B c D_{1} E F$ & $\alpha_{3}(1-\gamma)$ & $\mu_{3}$ \\
\hline 14 & $A B C D_{1} E F$ & $A B C d E F$ & $\alpha_{7}(1-\gamma)$ & $\mu_{7}$ \\
\hline & $A B C D_{1} E F$ & $A B C D_{1} E f$ & $\alpha_{6}(1-\gamma)$ & $\mu_{6}$ \\
\hline & & & & \\
\hline & & & & \\
\hline & & & & \\
\hline
\end{tabular}

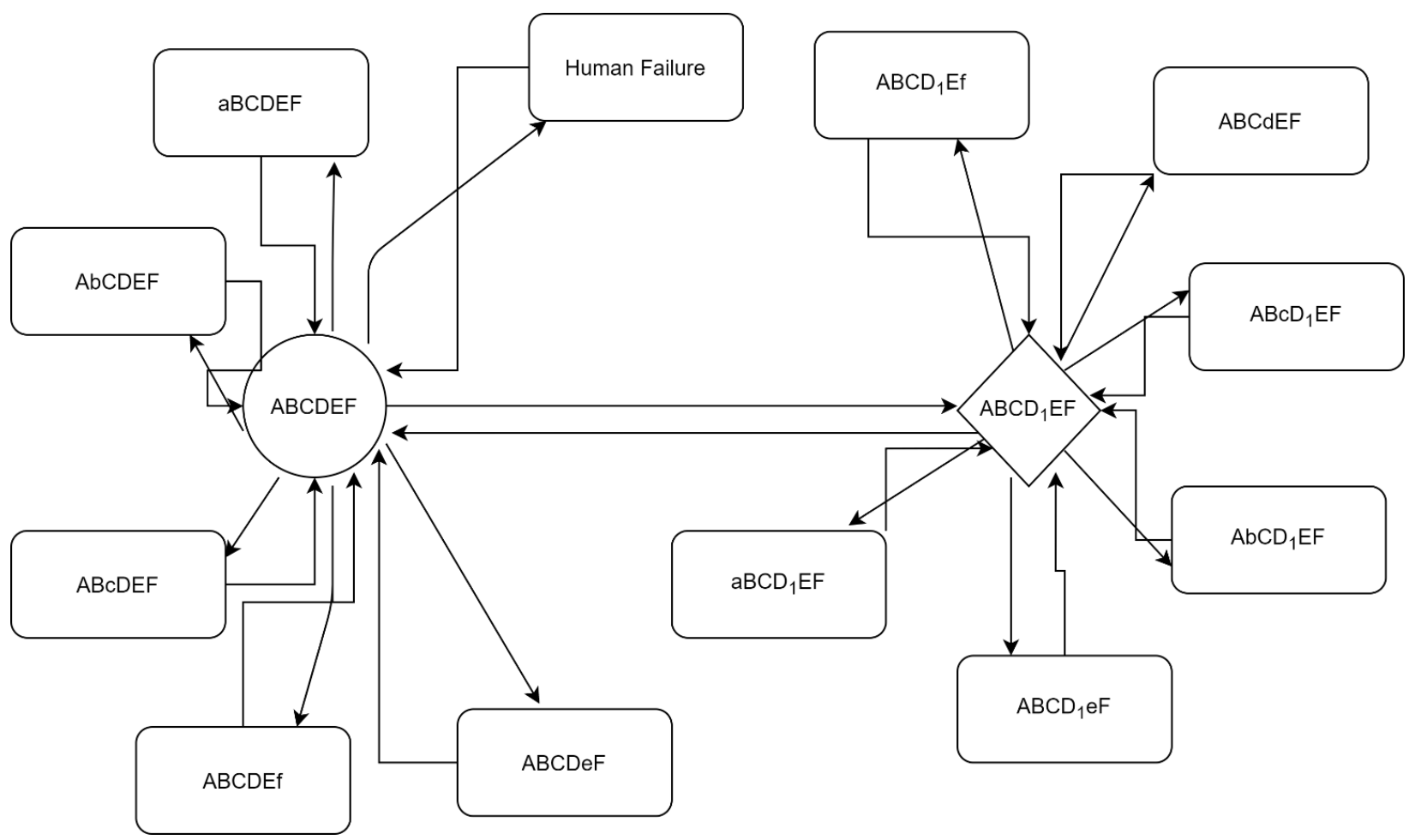

Fig.-1: State progression diagram of the system 


\section{ASSUMPTIONS}

a) No waiting time between failure and repair

b) Systems works as new after repair with full capacity

c) Failure and repair rates are exponentially distributed, and no simultaneous failures occurs.

d) Human failure can occur at the initial state.

\section{NOTATIONS}

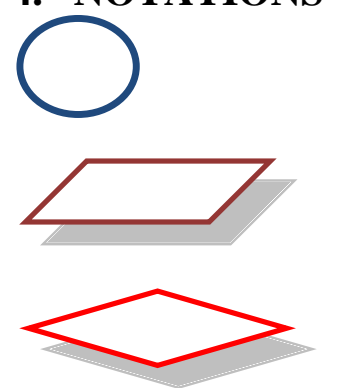
$A, B, C, D, E, F$
$a, b, c, d, e, f$
$D_{1}$
$\alpha_{i}(1 \leq i \leq 7)$

System works with full
capacity

Subsystem works with reduced capacity

Subsystem is in failure state

All subsystems are working with full capacity

Subsystem has failed

Partial operative state

Respectively failure rates in subsystems

$$
A, B, C, D, E, F, D_{1}
$$

Human failure and treatment rate

Respectively repair rate in subsystems

$$
A, B, C, D, E, F, D_{1}
$$

Probability that system is working with full capacity
$P_{i}(t),(i=1$,
Probability of subsystem on $\mathrm{i}^{\text {th }}$ state at time $\mathrm{t}$
$S_{i},(i=1,2$,
State of the subsystem
$\gamma$
Represent the coverage factor

\section{MATHEMATICAL MODELING OF THE SYSTEM}

A mathematical model for a cement manufacturing plant has been formulated using Markov birth-death process. The following Chapman-Kolmogorov equations have been derived:

$$
\begin{aligned}
& P_{1}(t+\Delta t)=\left[1-\left(\alpha_{1}(1-\gamma)+\alpha_{2}(1-\gamma)+\alpha_{3}(1-\gamma)+\alpha_{4}\right.\right. \\
& \left.\left.\gamma+\alpha_{5}(1-\gamma)+\alpha_{6}(1-\gamma)+\alpha_{H}(1-\gamma)\right)\right] \Delta t P_{1}(t) \\
& +\mu_{1} \Delta t P_{3}(t)+\mu_{2} \Delta t P_{4}(t)+\mu_{3} \Delta t P_{5}(t)+ \\
& \Delta t \mu_{6} P_{7}(t)+\Delta t \mu_{5} P_{6}(t)+\Delta t \mu_{H} P_{8}(t)+\Delta t \mu_{4} P_{2}(t) \\
& P_{1}(t+\Delta t)-P_{1}(t)+\left[\alpha_{1}(1-\gamma)+\alpha_{2}(1-\gamma)+\alpha_{3}\right. \\
& \left.(1-\gamma)+\alpha_{4} \gamma+\alpha_{5}(1-\gamma)+\alpha_{6}(1-\gamma)+\alpha_{H}(1-\gamma)\right] \Delta t P_{1}(t) \\
& =\mu_{1} \Delta t P_{3}(t)+\mu_{2} \Delta t P_{4}(t)+\mu_{3} \Delta t P_{5}(t)+ \\
& \Delta t \mu_{6} P_{7}(t)+\Delta t \mu_{5} P_{6}(t)+\Delta t \mu_{H} P_{8}(t)+\Delta t \mu_{4} P_{2}(t)
\end{aligned}
$$

Dividing equation $(*)$ both sides by $\Delta t$, we get

$$
\begin{aligned}
& \frac{P_{1}(t+\Delta t)-P_{1}(t)}{\Delta t}+\left[\alpha_{1}(1-\gamma)+\alpha_{2}(1-\gamma)+\right. \\
& \alpha_{3}(1-\gamma)+\alpha_{4} \gamma+\alpha_{5}(1-\gamma)+\alpha_{6}(1-\gamma)+ \\
& \left.\alpha_{H}(1-\gamma)\right] P_{1}(t)=\mu_{1} P_{3}(t)+\mu_{2} P_{4}(t)+\mu_{3} \\
& P_{5}(t)+\mu_{6} P_{7}(t)+\mu_{5} P_{6}(t)+\mu_{H} P_{8}(t)+\mu_{4} P_{2}(t)
\end{aligned}
$$

Taking limit $\Delta t \rightarrow 0$ on equation (**), we obtained

$$
\begin{aligned}
& \frac{d P_{1}(t)}{d t}+\left[\alpha_{1}(1-\gamma)+\alpha_{2}(1-\gamma)+\alpha_{3}(1-\gamma)+\alpha_{4} \gamma\right. \\
& \left.+\alpha_{5}(1-\gamma)+\alpha_{6}(1-\gamma)+\alpha_{H}(1-\gamma)\right] P_{1}(t) \\
& =\mu_{1} P_{3}(t)+\mu_{2} P_{4}(t)+\mu_{3} P_{5}(t)+ \\
& \mu_{6} P_{7}(t)+\mu_{5} P_{6}(t)+\mu_{H} P_{8}(t)+\mu_{4} P_{2}(t)
\end{aligned}
$$




$$
\begin{aligned}
& \frac{d P_{2}(t)}{d t}+\left[\alpha_{1}(1-\gamma)+\alpha_{2}(1-\gamma)+\alpha_{3}(1-\gamma)+\alpha_{7}(1-\gamma)\right. \\
& \left.+\alpha_{5}(1-\gamma)+\alpha_{6}(1-\gamma)+\alpha_{H}(1-\gamma)+\mu_{4}\right] P_{2}(t) \\
& \quad=\alpha_{4} P_{1}(t)+\mu_{1} P_{10}(t)+\mu_{2} P_{9}(t)+\mu_{3} \\
& P_{12}(t)+\mu_{7} P_{13}(t)+\mu_{5} P_{11}(t)+\mu_{H} P_{8}(t)+\mu_{6} P_{14}(t) \\
& \frac{d P_{3}(t)}{d t}+\mu_{1} P_{3}(t)=\alpha_{1}(1-\gamma) P_{1}(t) \\
& \frac{d P_{4}(t)}{d t}+\mu_{2} P_{4}(t)=\alpha_{2}(1-\gamma) P_{1}(t) \\
& \frac{d P_{5}(t)}{d t}+\mu_{3} P_{5}(t)=\alpha_{3}(1-\gamma) P_{1}(t) \\
& \frac{d P_{6}(t)}{d t}+\mu_{5} P_{6}(t)=\alpha_{5}(1-\gamma) P_{1}(t) \\
& \frac{d P_{14}(t)}{d t}+\mu_{6} P_{14}(t)=\alpha_{6}(1-\gamma) P_{2}(t) \\
& \frac{d P_{7}(t)}{d t}+\mu_{6} P_{7}(t)=\alpha_{6}(1-\gamma) P_{1}(t) \\
& \frac{d P_{12}(t)}{d t}+\mu_{3} P_{12}(t)=\alpha_{3}(1-\gamma) P_{2}(t) \\
& \frac{d P_{8}(t)}{d t}+\mu_{H} P_{8}(t)=\alpha_{H}(1-\gamma) P_{1}(t) \\
& \frac{d P_{9}(t)}{d t}+\mu_{2} P_{9}(t)=\alpha_{2}(1-\gamma) P_{2}(t) \\
& \frac{d t}{d t}+\mu_{1} P_{10}(t)=\alpha_{1}(1-\gamma) P_{2}(t)
\end{aligned}
$$

with initial conditions:

$$
P_{i}(0)= \begin{cases}1, & \text { if } i=1 \\ 0, & \text { if } i \neq 1\end{cases}
$$

Thus, the system availability and profit have been given by 
$A(t)=P_{1}(t)+P_{2}(t)$

$P_{\text {down }}(t)=\sum_{i=3}^{14} P_{i}(t)$

$\operatorname{Pr} o f i t=K A(t)-E P_{\text {down }}(t)$

Where $\mathrm{K}=10000$ and $\mathrm{E}=100$.

\section{PERFORMANCE ANALYSIS}

In this section, numerical analysis has been done for a cement manufacturing plant using equation (18) for a particular set of failure and repair rates corresponding to various values of coverage factor.

Impact of coverage factor $(\gamma)$ on profit and availability of cement manufacturing plant

Numerical results for availability and profit function have been derived for a particular set of failure and repair rates of various subsystems. The availability values of system for various values of coverage parameter has been derived and appended in table 2 . The fixed values of the parameters are as

Table 2: Impact of coverage factor $(\gamma)$ on profit and availability of cement manufacturing plant w.r.t. time

\begin{tabular}{r|ccccccccc}
\hline Time & $\gamma=0$ & $\gamma=0.1$ & $\gamma=0.2$ & $\gamma=0.4$ & $\gamma=0.5$ & $\gamma=0.6$ & $\gamma=0.8$ & $\gamma=0.9$ & $\gamma=1$ \\
\hline 40 & 0.886 & 0.896 & 0.9078 & 0.9276 & 0.9376 & 0.9486 & 0.9686 & 0.9796 & 0.9904 \\
80 & 0.886 & 0.896 & 0.9078 & 0.9276 & 0.9376 & 0.9486 & 0.9686 & 0.9796 & 0.9904 \\
120 & 0.886 & 0.896 & 0.9058 & 0.9268 & 0.9368 & 0.9478 & 0.99686 & 0.99796 & 0.9904 \\
160 & 0.886 & 0.896 & 0.9058 & 0.9268 & 0.9368 & 0.9478 & 0.9686 & 0.9796 & 0.9904 \\
200 & 0.886 & 0.896 & 0.9058 & 0.9268 & 0.9368 & 0.9478 & 0.9686 & 0.9796 & 0.9904 \\
\hline
\end{tabular}

Influence of raw mill failure and repair rate on availability and profit of cement manufacturing plant has been investigated for various values of failure and repair parameters. It is observed from table $3 \& 4$ that availability and profit increases with respect to increase of repair rate while decrease with respect to failure rates.

Table 3: Influence of failure rate $\left(\alpha_{1}\right)$ and repair rate $\left(\mu_{1}\right)$ of Raw mill on system's availability with respect to time

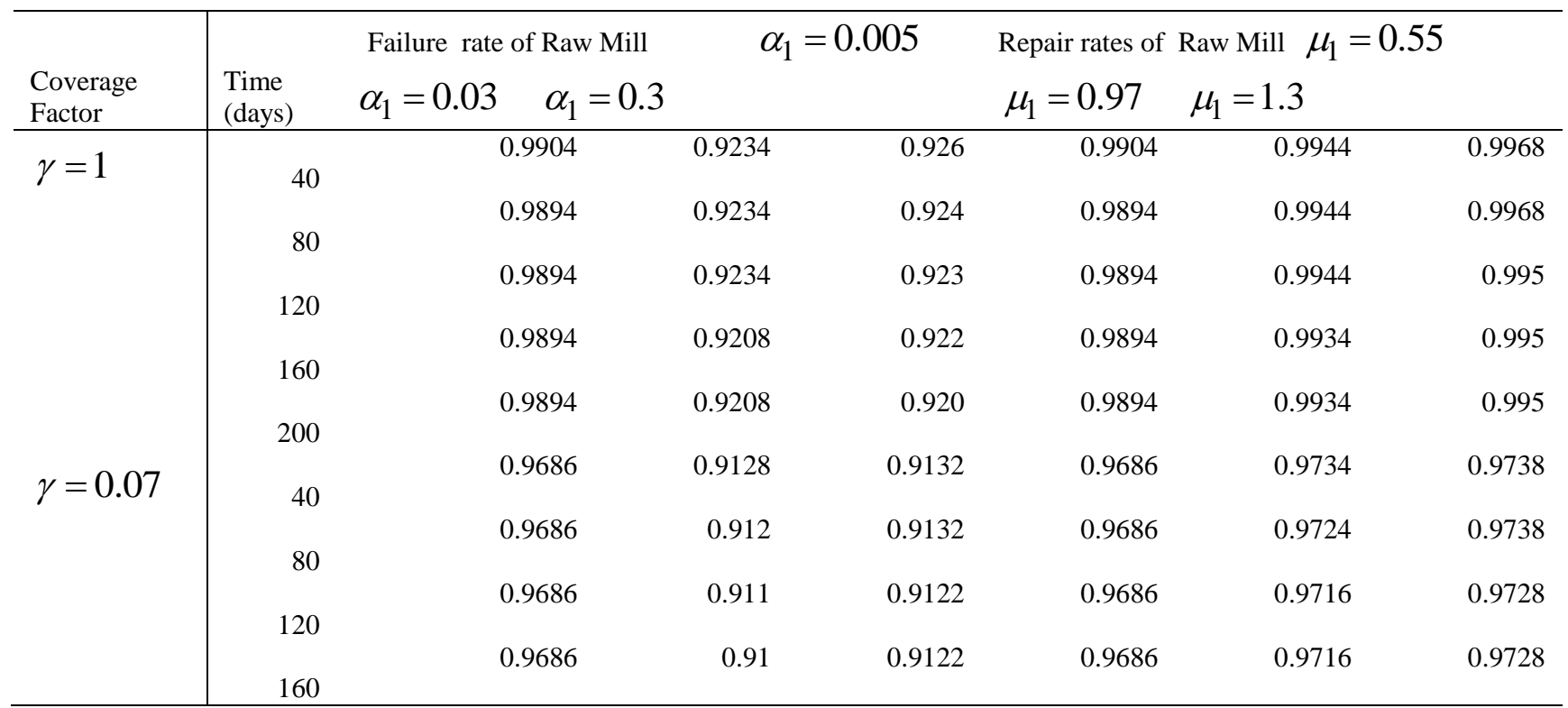




\begin{tabular}{|c|c|c|c|c|c|c|c|}
\hline & & 0.9686 & 0.91 & 0.9114 & 0.9686 & 0.9716 & 0.9728 \\
\hline \multirow{5}{*}{$\gamma=0.4$} & 200 & 0.9376 & 0.906 & 0.902 & 0.9376 & 0.94 & 0.94 \\
\hline & Q0 & 0.9376 & 0.90 & 0.902 & 0.9376 & 0.9392 & 0.939 \\
\hline & ov & 0.9368 & 0.90 & 0.902 & 0.9368 & 0.9392 & 0.939 \\
\hline & 120 & 0.9368 & 0.90 & 0.902 & 0.9368 & 0.9384 & 0.939 \\
\hline & 000 & 0.9368 & 0.90 & 0.90 & 0.9368 & 0.9374 & 0.939 \\
\hline \multirow{5}{*}{$\gamma=0$} & 40 & 0.886 & 0.885 & 0.885 & 0.886 & 0.886 & 0.886 \\
\hline & & 0.886 & 0.885 & 0.885 & 0.886 & 0.886 & 0.886 \\
\hline & 120 & 0.886 & 0.884 & 0.884 & 0.886 & 0.886 & 0.886 \\
\hline & 160 & 0.886 & 0.884 & 0.884 & 0.886 & 0.886 & 0.886 \\
\hline & 200 & 0.886 & 0.884 & 0.884 & 0.886 & 0.886 & 0.886 \\
\hline
\end{tabular}

Table 4 : Influence of failure rate $\left(\alpha_{1}\right)$ and repair rate $\left(\mu_{1}\right)$ of Raw mill on system's profit with respect to time

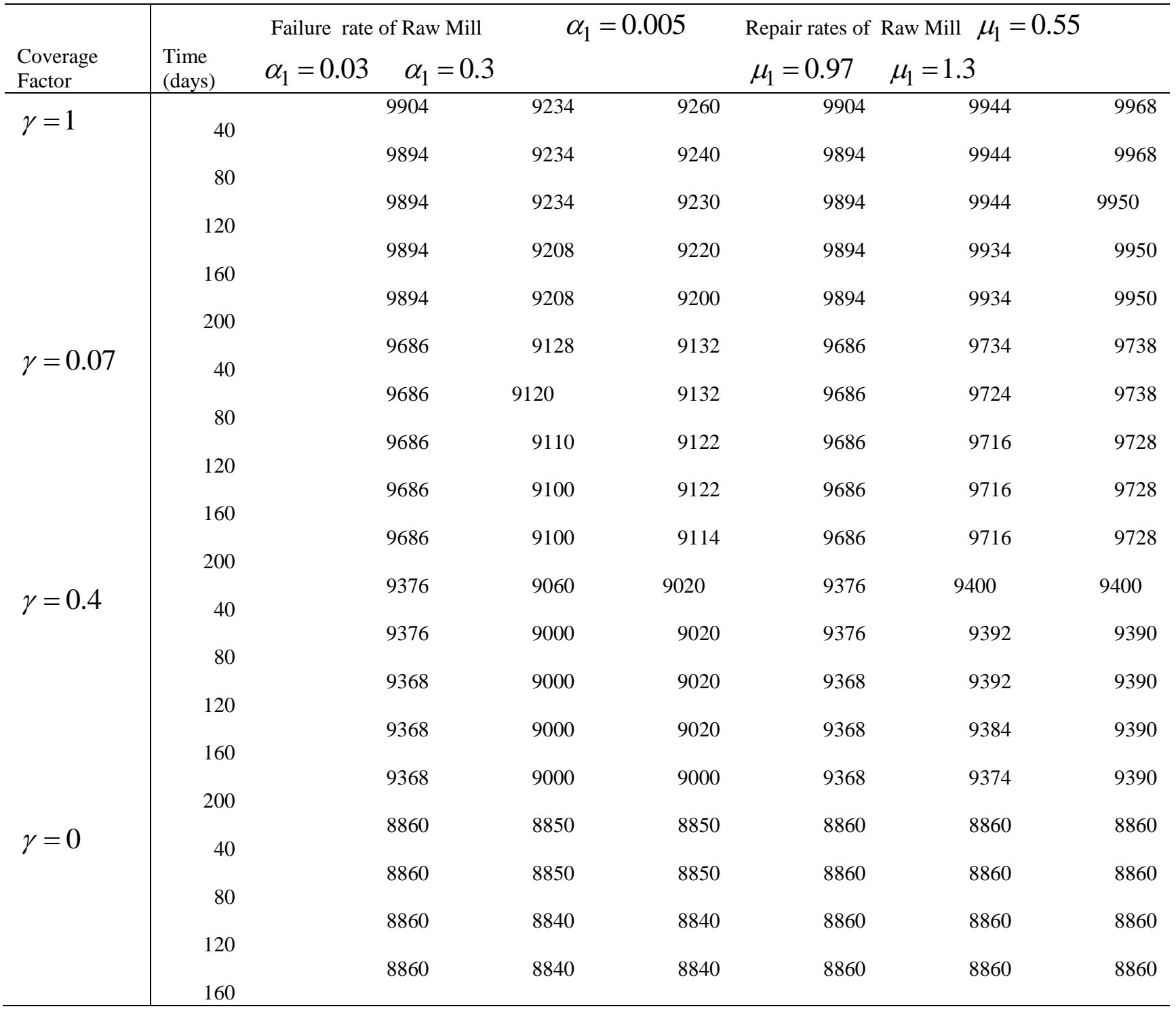




\begin{tabular}{l|llccccc}
\hline & 200 & 8860 & 8840 & 8840 & 8860 & 8860 & 8860 \\
\hline
\end{tabular}

Influence of coal mill failure and repair rate on availability and profit of cement manufacturing plant has been investigated for various values of failure and repair parameters. It is observed from table $5 \& 6$ that availability and profit increases with respect to increase of repair rate while decrease with respect to failure rates.

Table 5: Influence of failure rate $\left(\alpha_{2}\right)$ and repair rate $\left(\mu_{2}\right)$ of coal mill on system's availability with respect to time

\begin{tabular}{|c|c|c|c|c|c|c|c|c|}
\hline \multirow[b]{2}{*}{$\begin{array}{l}\text { Coverage } \\
\text { Factor }\end{array}$} & \multirow[b]{2}{*}{$\begin{array}{l}\text { Time } \\
\text { (days) }\end{array}$} & \multicolumn{2}{|c|}{ Failure rate of Coal Mill } & \multicolumn{2}{|c|}{$\alpha_{2}=0.002$} & \multicolumn{3}{|c|}{ Repair rates of Coal Mill } \\
\hline & & $\alpha_{2}=0.3$ & $\alpha_{2}=0.6$ & & & $\mu_{2}=0.81$ & $\mu_{2}=1.21$ & $\mu_{2}=1.91$ \\
\hline \multirow{7}{*}{$\gamma=1$} & $x_{0}=7$, & & 0.9904 & 0.9098 & 0.9702 & 0.9904 & 0.9906 & 0.9898 \\
\hline & & & 0.9894 & 0.9098 & 0.9692 & 0.9894 & 0.9906 & 0.9898 \\
\hline & 80 & & & & & & & \\
\hline & & & 0.9894 & 0.9098 & 0.9692 & 0.9894 & 0.9906 & 0.9898 \\
\hline & 120 & & 0.9894 & 0.9088 & 0.9692 & 0.9894 & 0.9896 & 0.9898 \\
\hline & 160 & & & & & & & \\
\hline & 200 & & 0.9894 & 0.9088 & 0.9692 & 0.9894 & 0.9888 & 0.9898 \\
\hline \multirow{8}{*}{$\gamma=0.07$} & 200 & & 0.9686 & 0.9004 & 0.8803 & 0.9686 & 0.969 & 0.9688 \\
\hline & 40 & & & & & & & \\
\hline & ( & & 0.9686 & 0.9004 & 0.8803 & 0.9686 & 0.968 & 0.9688 \\
\hline & 80 & & 0.9686 & 0.8996 & 08803 & 0.9686 & 0.968 & 0.9688 \\
\hline & 120 & & & & & & & 0.3000 \\
\hline & & & 0.9686 & 0.8986 & 0.8803 & 0.9686 & 0.967 & 0.9688 \\
\hline & 160 & & 0.0686 & 08086 & 08803 & 0.0686 & 0067 & 0.9688 \\
\hline & 200 & & & & & & 0.901 & 0.9000 \\
\hline \multirow{7}{*}{$\gamma=0.4$} & 40 & & 0.9376 & 0.8912 & 0.8256 & 0.9376 & 0.9378 & 0.937 \\
\hline & & & 0.9376 & 0.8912 & 0.8246 & 0.9376 & 0.9378 & 0.937 \\
\hline & 80 & & 09368 & 08912 & 08246 & 00368 & 00378 & 0.937 \\
\hline & 120 & & & & 0.0240 & 0.9500 & 0.9510 & 0.935 \\
\hline & & & 0.9368 & 0.8902 & 0.8246 & 0.9368 & 0.9378 & 0.937 \\
\hline & 160 & & 00368 & 08002 & 08246 & 00368 & 00378 & 0.037 \\
\hline & 200 & & & & & & 0.9570 & 1 \\
\hline \multirow{7}{*}{$\gamma=0$} & 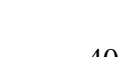 & & 0.886 & 0.885 & 0.85 & 0.886 & 0.886 & 0.886 \\
\hline & & & 0.886 & 0.885 & 0.85 & 0.886 & 0.886 & 0.886 \\
\hline & 80 & & & & & & & \\
\hline & & & 0.886 & 0.884 & 0.84 & 0.886 & 0.886 & 0.886 \\
\hline & 120 & & & & & & & \\
\hline & & & 0.886 & 0.884 & 0.84 & 0.886 & 0.886 & 0.886 \\
\hline & 200 & & 0.886 & 0.884 & 0.84 & 0.886 & 0.886 & 0.886 \\
\hline
\end{tabular}

Table 6: Influence of failure rate $\left(\alpha_{2}\right)$ and repair rate $\left(\mu_{2}\right)$ of coal mill on system's profit with respect to time

\begin{tabular}{l|ccccrrrr}
\hline & \multicolumn{3}{c}{ Failure rate of Coal Mill } & \multicolumn{2}{c}{$\alpha_{2}=0.002$} & \multicolumn{3}{c}{ Repair rates of Coal Mill } \\
$\begin{array}{l}\text { Coverage } \\
\text { Factor }\end{array}$ & $\begin{array}{l}\text { Time } \\
\text { (days) }\end{array}$ & $\alpha_{2}=0.3$ & $\alpha_{2}=0.6$ & & & $\mu_{2}=0.81$ & $\mu_{2}=1.21$ & $\mu_{2}=1.91$ \\
\hline$\gamma=1$ & 40 & 9904 & 9098 & 9702 & 9904 & 9906 & 9898 \\
& 40 & 9894 & 9098 & 9692 & 9894 & 9906 & 9898
\end{tabular}




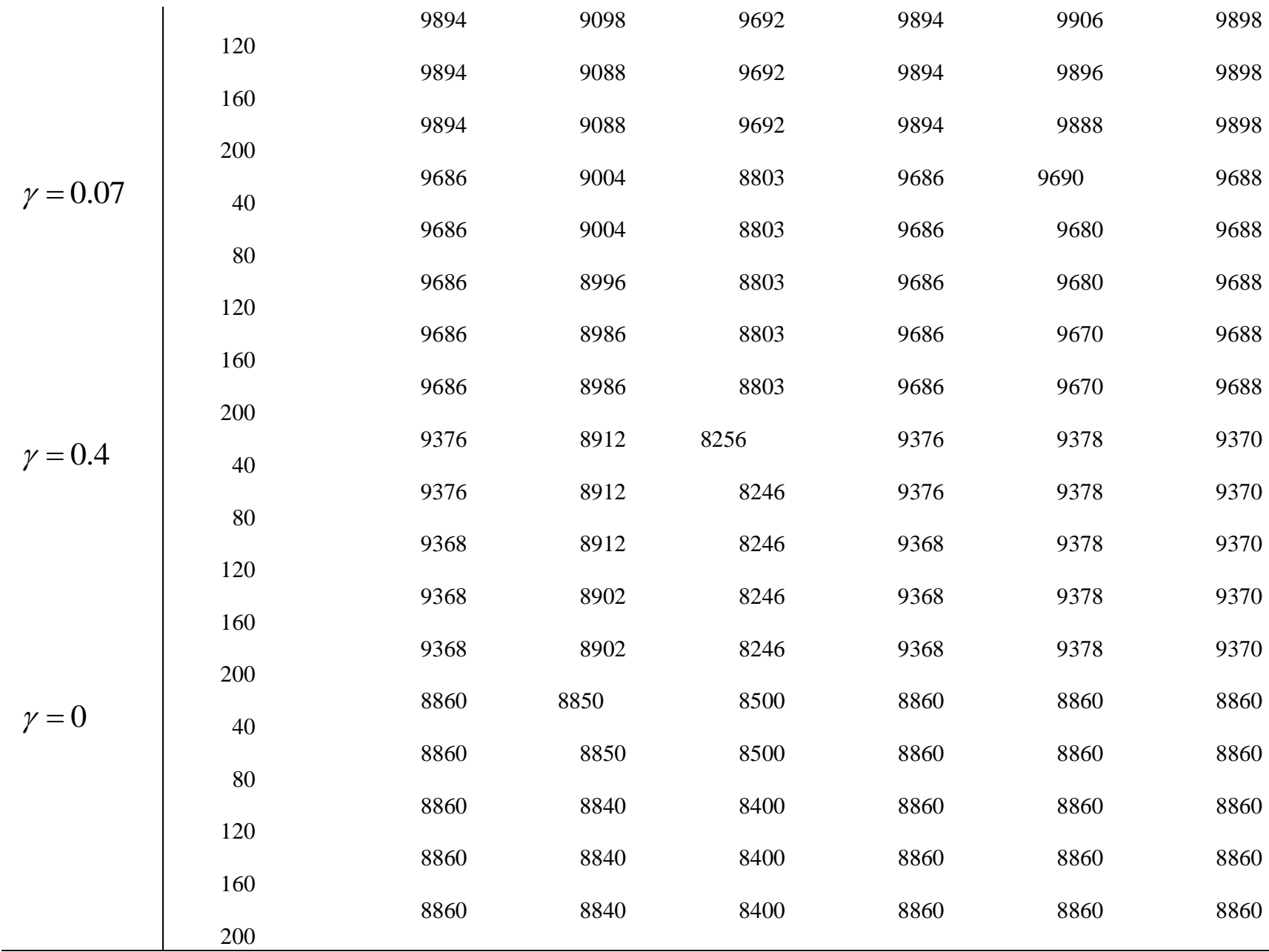

Influence of preheater's failure and repair rate on availability and profit of cement manufacturing plant has been investigated for various values of failure and repair parameters. It is observed from table $7 \& 8$ that availability and profit increases with respect to increase of repair rate while decrease with respect to failure rates.

Table 7: Influence of failure rate $\left(\alpha_{3}\right)$ and repair rate $\left(\mu_{3}\right)$ of Preheater on system's availability with respect to time

\begin{tabular}{|c|c|c|c|c|c|c|c|c|}
\hline \multirow[b]{2}{*}{$\begin{array}{l}\text { Coverage } \\
\text { Factor }\end{array}$} & \multirow[b]{2}{*}{$\begin{array}{l}\text { Time } \\
\text { (days) }\end{array}$} & \multicolumn{2}{|c|}{ Failure rates of Preheater } & \multicolumn{2}{|c|}{$\alpha_{3}=0.001$} & \multicolumn{3}{|c|}{ Repair rates of Preheater } \\
\hline & & $\alpha_{3}=0.3$ & $\alpha_{3}=0.9$ & & & $\mu_{3}=0.6$ & $\mu_{3}=0.9$ & $\mu_{3}=1.6$ \\
\hline \multirow[t]{5}{*}{$\gamma=1$} & 40 & & 0.9904 & 0.9904 & 0.9904 & 0.9904 & 0.9904 & 0.9904 \\
\hline & & & 0.9894 & 0.9894 & 0.9894 & 0.9894 & 0.9894 & 0.9894 \\
\hline & $\begin{array}{r}80 \\
120\end{array}$ & & 0.9894 & 0.9894 & 0.9894 & 0.9894 & 0.9894 & 0.9894 \\
\hline & 160 & & 0.9894 & 0.9894 & 0.9894 & 0.9894 & 0.9894 & 0.9894 \\
\hline & 200 & & 0.9894 & 0.9894 & 0.9894 & 0.9894 & 0.9894 & 0.9894 \\
\hline \multirow[t]{4}{*}{$\gamma=0.07$} & 40 & & 0.9686 & 0.8786 & 0.6844 & 0.9686 & 0.9746 & 0.9756 \\
\hline & 80 & & 0.9686 & 0.8786 & 0.6844 & 0.9686 & 0.9746 & 0.9756 \\
\hline & 120 & & 0.9686 & 0.8786 & 0.6834 & 0.9686 & 0.9736 & 0.9746 \\
\hline & 160 & & 0.9686 & 0.8786 & 0.6834 & 0.9686 & 0.9736 & 0.9746 \\
\hline
\end{tabular}




\begin{tabular}{|c|c|c|c|c|c|c|c|}
\hline & & 0.9686 & 0.8786 & 0.6834 & 0.9686 & 0.9736 & 0.9746 \\
\hline \multirow{5}{*}{$\gamma=0.4$} & & 0.9376 & 0.7522 & 0.4976 & 0.9376 & 0.9528 & 0.9568 \\
\hline & & 0.9376 & 0.7522 & 0.4964 & 0.9376 & 0.9518 & 0.9568 \\
\hline & & 0.9368 & 0.7522 & 0.4964 & 0.9368 & 0.9518 & 0.9558 \\
\hline & & 0.9368 & 0.7522 & 0.4964 & 0.9368 & 0.9518 & 0.9558 \\
\hline & & 0.9368 & 0.7522 & 0.4964 & 0.9368 & 0.9518 & 0.9558 \\
\hline \multirow{7}{*}{$\gamma=0$} & 200 & 0.886 & 0.594 & 0.371 & 0.886 & 0.914 & 0.924 \\
\hline & 40 & & & & & & \\
\hline & & 0.886 & 0.594 & 0.371 & 0.886 & 0.914 & 0.924 \\
\hline & & 0.886 & 0.594 & 0.371 & 0.886 & 0.914 & 0.924 \\
\hline & 12 & 0.886 & 0.594 & 0.37 & 0.886 & 0.914 & 0.924 \\
\hline & 160 & 0886 & 0504 & 037 & 0.896 & 0914 & 0024 \\
\hline & 200 & & & & & & \\
\hline
\end{tabular}

Table 8: Influence of failure rate $\left(\alpha_{3}\right)$ and repair rate $\left(\mu_{3}\right)$ of Preheater on system's profit with respect to time

\begin{tabular}{|c|c|c|c|c|c|c|c|c|}
\hline \multirow[b]{2}{*}{$\begin{array}{l}\text { Coverage } \\
\text { Factor }\end{array}$} & \multirow[b]{2}{*}{$\begin{array}{l}\text { Time } \\
\text { (days) }\end{array}$} & \multicolumn{2}{|c|}{ Failure rates of Preheater } & \multicolumn{2}{|c|}{$\alpha_{3}=0.001$} & \multicolumn{3}{|c|}{ Repair rates of Preheater } \\
\hline & & $\alpha_{3}=0.3$ & $\alpha_{3}=0.9$ & & & $\mu_{3}=0.6$ & $\mu_{3}=0.9$ & $\mu_{3}=1.6$ \\
\hline \multirow{7}{*}{$\gamma=1$} & (c) & & 9904 & 9904 & 9904 & 9904 & 9904 & 9904 \\
\hline & & & 9894 & 9894 & 9894 & 9894 & 9894 & 9894 \\
\hline & 80 & & & & & & & \\
\hline & 100 & & 9894 & 9894 & 9894 & 9894 & 9894 & 9894 \\
\hline & & & 9894 & 9894 & 9894 & 9894 & 9894 & 9894 \\
\hline & 160 & & & & & & & \\
\hline & & & 9894 & 9894 & 9894 & 9894 & 9894 & 9894 \\
\hline \multirow{8}{*}{$\gamma=0.07$} & 200 & & & 0706 & 604 & 0686 & & \\
\hline & 40 & & 9686 & 8786 & 6844 & 9686 & 9746 & 9756 \\
\hline & & & 9686 & 8786 & 6844 & 9686 & 9746 & 9756 \\
\hline & 80 & & 0686 & 8786 & 6834 & 0686 & 0736 & 0746 \\
\hline & 120 & & & & & & & \\
\hline & & & 9686 & 8786 & 6834 & 9686 & 9736 & 9746 \\
\hline & 160 & & 9686 & 8786 & 6834 & 9686 & 0736 & 0746 \\
\hline & 200 & & & & & & & \\
\hline \multirow[t]{7}{*}{$\gamma=0.4$} & 40 & & 9376 & 7522 & 4976 & 9376 & 9528 & 9568 \\
\hline & & & 9376 & 7522 & 4964 & 9376 & 9518 & 9568 \\
\hline & 80 & & & & & & & \\
\hline & 120 & & 9368 & 7522 & 4964 & 9368 & 9518 & 9558 \\
\hline & & & 9368 & 7522 & 4964 & 9368 & 9518 & 9558 \\
\hline & 160 & & 0260 & 7500 & 1064 & & & \\
\hline & 200 & & & $1 J \angle 2$ & 4904 & 9300 & 9010 & 9558 \\
\hline \multirow{5}{*}{$\gamma=0$} & & & 8860 & 5940 & 3710 & 8860 & 9140 & 9240 \\
\hline & & & 8860 & 5040 & 3710 & 8860 & 0140 & 0240 \\
\hline & 80 & & & & & & & \\
\hline & & & 8860 & 5940 & 3710 & 8860 & 9140 & 9240 \\
\hline & 160 & & 8860 & 5940 & 3710 & 8860 & 9140 & 9240 \\
\hline
\end{tabular}


Influence of klin's failure and repair rate on availability and profit of cement manufacturing plant has been investigated for various values of failure and repair parameters. It is observed from table 9 and 10 that availability and profit increases with respect to increase of repair rate while decrease with respect to failure rates.

Table 9: Influence of failure rate ( $\left.\alpha_{4}\right)$ and repair rate $\left(\mu_{4}\right)$ of Klin on system's availability with respect to time

\begin{tabular}{|c|c|c|c|c|c|c|c|}
\hline \multirow[b]{2}{*}{$\begin{array}{l}\text { Coverage } \\
\text { Factor }\end{array}$} & \multirow[b]{2}{*}{$\begin{array}{l}\text { Time } \\
\text { (days) }\end{array}$} & \multicolumn{3}{|c|}{ Failure rates of Klin } & \multicolumn{2}{|c|}{ Repair rates of Klin } & \multirow[b]{2}{*}{$\mu_{4}=1.3$} \\
\hline & & $\alpha_{4}=0.0012$ & $\alpha_{4}=0.5$ & $\alpha_{4}=0.83$ & $\mu_{4}=0.65$ & $\mu_{4}=0.9$ & \\
\hline \multirow{6}{*}{$\gamma=1$} & 40 & 0.9904 & 0.9904 & 0.9904 & 0.9904 & 0.9904 & 0.9904 \\
\hline & & 0.9894 & 0.9894 & 0.9894 & 0.9894 & 0.9894 & 0.9894 \\
\hline & & 0.9894 & 0.9894 & 0.9894 & 0.9894 & 0.9894 & 0.9894 \\
\hline & 120 & 0.9894 & 0.9894 & 0.9894 & 0.9894 & 0.9894 & 0.9894 \\
\hline & 160 & & & & & & \\
\hline & & 0.9894 & 0.9894 & 0.9894 & 0.9894 & 0.9894 & 0.9894 \\
\hline \multirow{8}{*}{$\gamma=0.07$} & 200 & 0.9686 & 0.8386 & 0.6616 & 0.9686 & 0.9686 & 0.9686 \\
\hline & 40 & & & & & & \\
\hline & & 0.9686 & 0.8378 & 0.6616 & 0.9686 & 0.9686 & 0.9686 \\
\hline & 80 & 0.9686 & 0.837 & 0.6616 & 0.9686 & 0.9686 & 0.9686 \\
\hline & 120 & & & & & & \\
\hline & & 0.9686 & 0.837 & 0.6616 & 0.9686 & 0.9686 & 0.9686 \\
\hline & 100 & 0.9686 & 0.837 & 0.6616 & 0.9686 & 0.9686 & 0.9686 \\
\hline & 200 & & & & & & \\
\hline \multirow{6}{*}{$\gamma=0.4$} & 40 & 0.9376 & 0.6444 & 0.578 & 0.9376 & 0.9378 & 0.9378 \\
\hline & & 0.9376 & 0.6444 & 0.578 & 0.9376 & 0.9378 & 0.9378 \\
\hline & 80 & 0.9368 & 0.6434 & 0.578 & 0.9368 & 0.9368 & 0.9368 \\
\hline & 120 & 0.9368 & 0.6434 & 0.578 & 0.9368 & 0.9368 & 0.9368 \\
\hline & & 0.9368 & 0.6434 & 0.578 & 0.9368 & 0.9368 & 0.9368 \\
\hline & 200 & & & & & & \\
\hline \multirow{7}{*}{$\gamma=0$} & & 0.886 & 0.507 & 0.368 & 0.886 & 0.886 & 0.887 \\
\hline & & 0.886 & 0.507 & 0.368 & 0.886 & 0.886 & 0.887 \\
\hline & 80 & & & & & & \\
\hline & & 0.886 & 0.506 & 0.368 & 0.886 & 0.886 & 0.886 \\
\hline & & 0886 & 0506 & 0268 & 0896 & 0896 & 0886 \\
\hline & 160 & 0.000 & 0.300 & 0.500 & 0.000 & 0.886 & 0.880 \\
\hline & 200 & 0.886 & 0.506 & 0.368 & 0.886 & 0.886 & 0.886 \\
\hline
\end{tabular}

Table 10: Influence of failure rate $\left(\alpha_{4}\right)$ and repair rate $\left(\mu_{4}\right)$ of Klin on system's profit with respect to time

\begin{tabular}{|c|c|c|c|c|c|c|c|}
\hline \multirow[b]{2}{*}{$\begin{array}{l}\text { Coverage } \\
\text { Factor }\end{array}$} & \multirow[b]{2}{*}{$\begin{array}{l}\text { Time } \\
\text { (days) }\end{array}$} & \multicolumn{3}{|c|}{ Failure rates of Klin } & \multicolumn{2}{|c|}{ Repair rates of Klin } & \multirow[b]{2}{*}{$\mu_{4}=1.3$} \\
\hline & & $\alpha_{4}=0.0012$ & $\alpha_{4}=0.5$ & $\alpha_{4}=0.83$ & $\mu_{4}=0.65$ & $\mu_{4}=0.9$ & \\
\hline \multirow[t]{3}{*}{$\gamma=1$} & 40 & 9904 & 9904 & 9904 & 9904 & 9904 & 9904 \\
\hline & & 9894 & 9894 & 9894 & 9894 & 9894 & 9894 \\
\hline & $\begin{array}{r}80 \\
120\end{array}$ & 9894 & 9894 & 9894 & 9894 & 9894 & 9894 \\
\hline
\end{tabular}




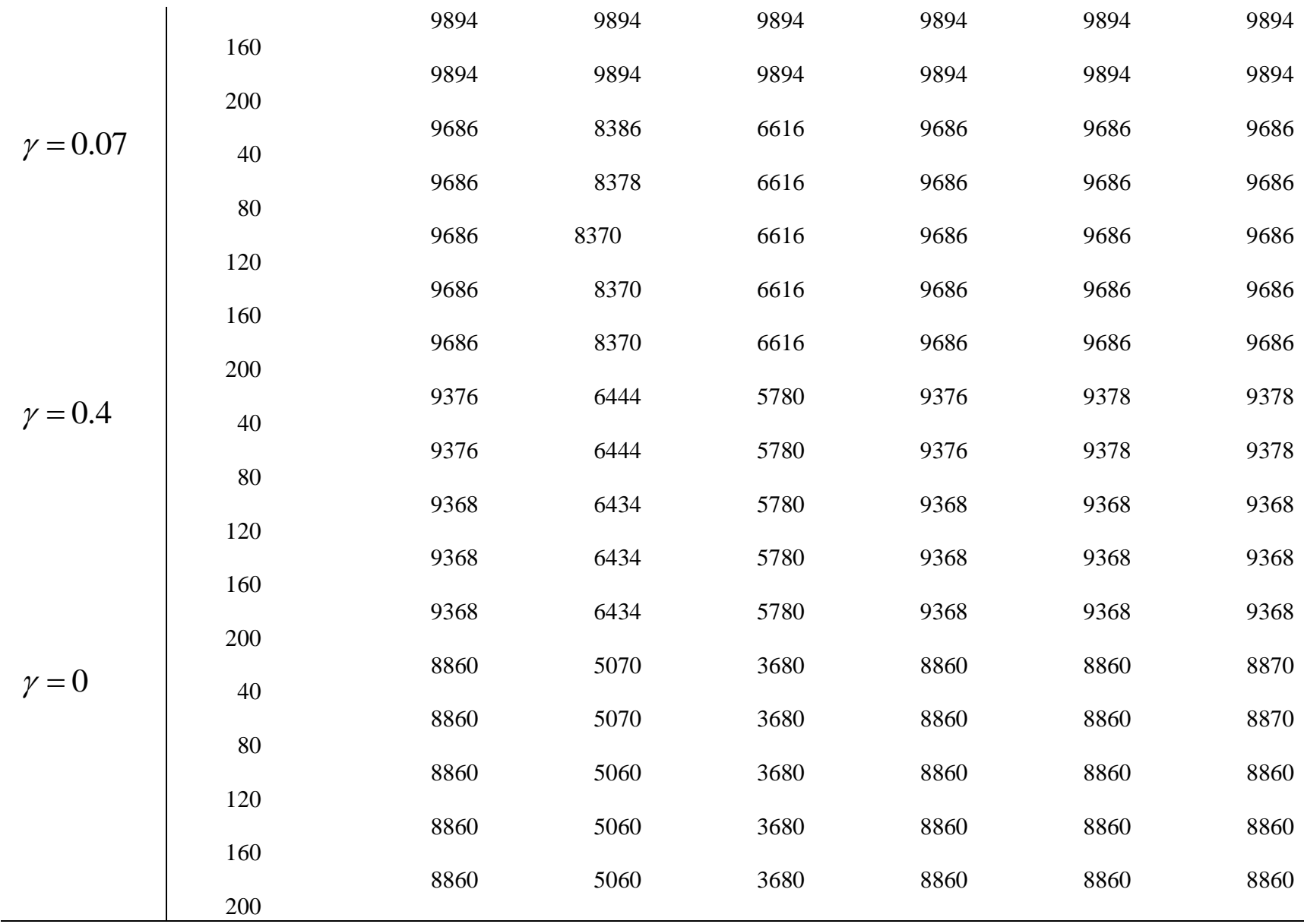

Influence of cooler's failure and repair rate on availability and profit of cement manufacturing plant has been investigated for various values of failure and repair parameters. It is observed from table 11-12 that availability and profit increases with respect to increase of repair rate while decrease with respect to failure rates.

Table 11: Influence of failure rate $\left(\alpha_{5}\right)$ and repair rate $\left(\mu_{5}\right)$ of cooler on system's availability with respect to time

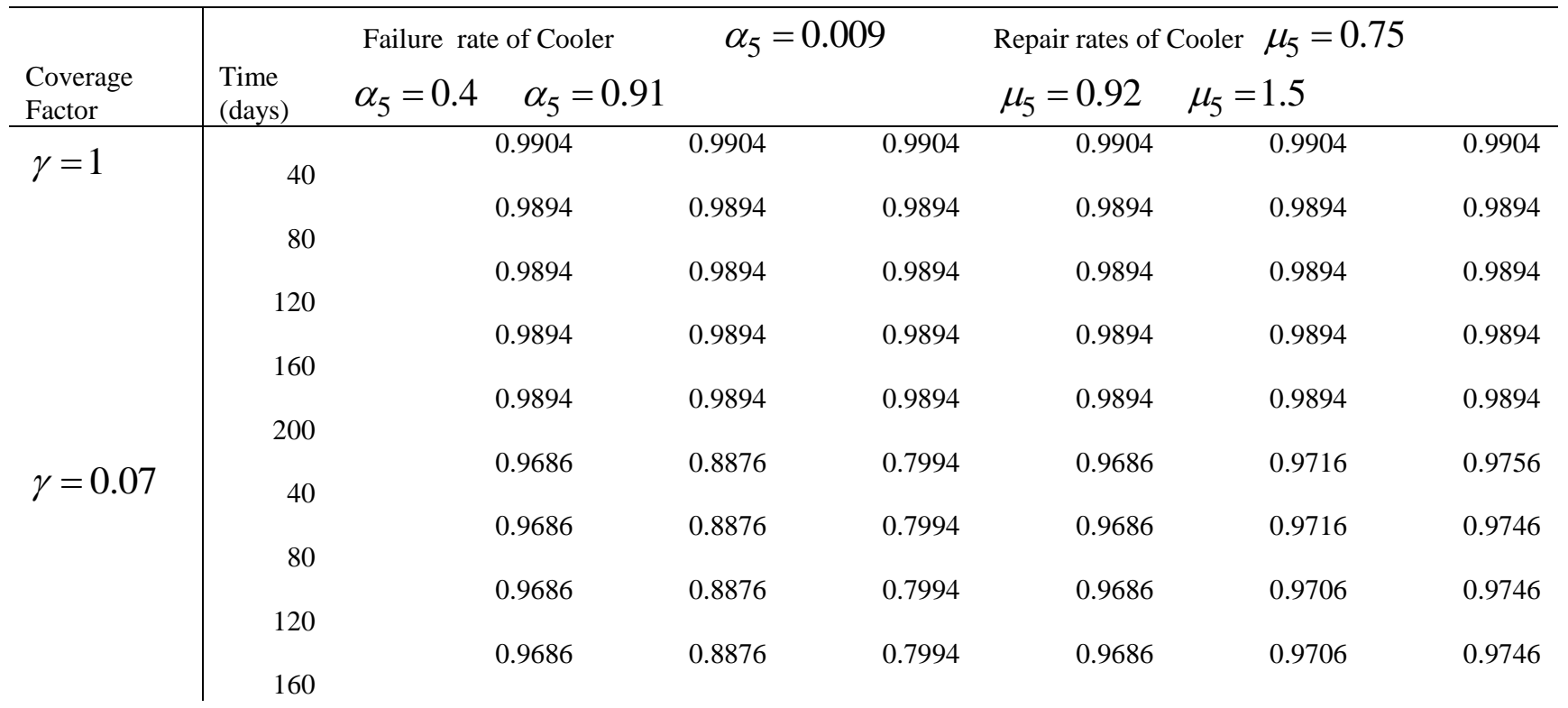




\begin{tabular}{|c|c|c|c|c|c|c|c|}
\hline & & 0.9686 & 0.8876 & 0.7994 & 0.9686 & 0.9706 & 0.9746 \\
\hline \multirow{5}{*}{$\gamma=0.4$} & & 0.9376 & 0.627 & 0.5708 & 0.9376 & 0.9458 & 0.9538 \\
\hline & & 0.9376 & 0.627 & 0.5698 & 0.9376 & 0.9458 & 0.9538 \\
\hline & & 0.9368 & 0.6262 & 0.5698 & 0.9368 & 0.9458 & 0.9538 \\
\hline & & 0.9368 & 0.6262 & 0.5698 & 0.9368 & 0.9458 & 0.9538 \\
\hline & & 0.9368 & 0.6262 & 0.5698 & 0.9368 & 0.9458 & 0.9538 \\
\hline \multirow{7}{*}{$\gamma=0$} & 200 & 0.886 & 0.670 & 0.457 & 0.886 & 0.903 & 0.919 \\
\hline & 40 & & 0660 & 0457 & 0.006 & 2002 & 0010 \\
\hline & 80 & 0.886 & 0.669 & $0.45 /$ & 0.886 & 0.903 & 0.919 \\
\hline & & 0.886 & 0.669 & 0.457 & 0.886 & 0.903 & 0.919 \\
\hline & & 0.886 & 0.669 & 0.456 & 0.886 & 0.902 & 0.919 \\
\hline & & 0.886 & 0.669 & 0.456 & 0.886 & 0.902 & 0.919 \\
\hline & 200 & & & & & & \\
\hline
\end{tabular}

Table 12: Influence of failure rate $\left(\alpha_{5}\right)$ and repair rate $\left(\mu_{5}\right)$ of cooler on system's profit with respect to time

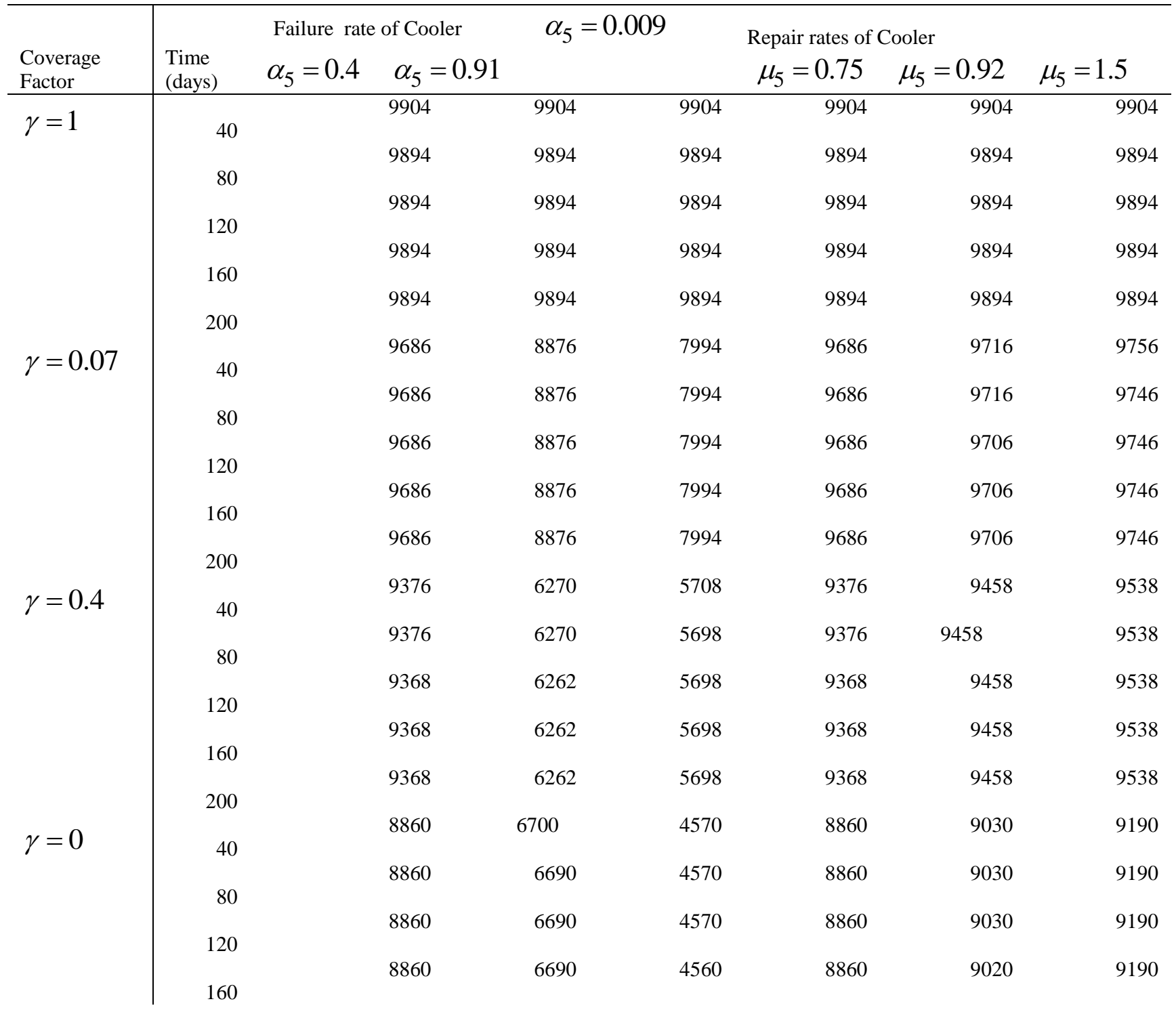


Influence of cement mill's failure and repair rate on availability and profit of cement manufacturing plant has been investigated for various values of failure and repair parameters. It is observed from table 13- 14 that availability and profit increases with respect to increase of repair rate while decrease with respect to failure rates.

Table 13: Influence of failure rate $\left(\alpha_{6}\right)$ and repair rate $\left(\mu_{6}\right)$ of cement mill on system's availability with respect to time

\begin{tabular}{|c|c|c|c|c|c|c|c|}
\hline \multirow[b]{2}{*}{$\begin{array}{l}\text { Coverage } \\
\text { Factor }\end{array}$} & \multirow[b]{2}{*}{$\begin{array}{l}\text { Time } \\
\text { (days) }\end{array}$} & \multicolumn{3}{|c|}{ Failure rates of cement mill } & \multicolumn{3}{|c|}{ Repair rates of cement mill } \\
\hline & & $\alpha_{6}=0.006$ & $\alpha_{4}=0.51$ & $\alpha_{4}=0.82$ & $\mu_{6}=0.65$ & $\mu_{6}=1.9$ & $\mu_{6}=2.3$ \\
\hline \multirow[t]{6}{*}{$\gamma=1$} & 40 & 0.9904 & 0.9904 & 0.9904 & 0.9904 & 0.9904 & 0.9904 \\
\hline & & 0.9894 & 0.9894 & 0.9894 & 0.9894 & 0.9894 & 0.9894 \\
\hline & & 0.9894 & 0.9894 & 0.9894 & 0.9894 & 0.9894 & 0.9894 \\
\hline & 120 & & & & & & \\
\hline & 160 & 0.9894 & 0.9894 & 0.9894 & 0.9894 & 0.9894 & 0.9894 \\
\hline & & 0.9894 & 0.9894 & 0.9894 & 0.9894 & 0.9894 & 0.9894 \\
\hline \multirow{7}{*}{$\gamma=0.07$} & & 0.9686 & 0.8386 & 0.6616 & 0.9686 & 0.9686 & 0.9686 \\
\hline & & 0.9686 & 0.8378 & 0.6616 & 0.9686 & 0.9686 & 0.9686 \\
\hline & 80 & & & & & & \\
\hline & & 0.9686 & 0.837 & 0.6616 & 0.9686 & 0.9686 & 0.9686 \\
\hline & & 0.9686 & 0.837 & 0.6616 & 0.9686 & 0.9686 & 0.9686 \\
\hline & & 0.9686 & 0.837 & 0.6616 & 0.9686 & 0.9686 & 0.9686 \\
\hline & 200 & & & & & & \\
\hline \multirow{6}{*}{$\gamma=0.4$} & 10 & 0.9376 & 0.6444 & 0.578 & 0.9376 & 0.9378 & 0.9378 \\
\hline & & 0.9376 & 0.6444 & 0.578 & 0.9376 & 0.9378 & 0.9378 \\
\hline & & 0.9368 & 0.6434 & 0.578 & 0.9368 & 0.9368 & 0.9368 \\
\hline & 120 & 0.9368 & 0.6434 & 0.578 & 0.9368 & 0.9368 & 0.9368 \\
\hline & 160 & & & & & & \\
\hline & & 0.9368 & 0.6434 & 0.578 & 0.9368 & 0.9368 & 0.9368 \\
\hline \multirow{7}{*}{$\gamma=0$} & 21 & 0.886 & 0.507 & 0.368 & 0.886 & 0.886 & 0.887 \\
\hline & & 0886 & 0.507 & 0368 & 0886 & 0.886 & 0.887 \\
\hline & 80 & & & & & & \\
\hline & & 0.886 & 0.506 & 0.368 & 0.886 & 0.886 & 0.886 \\
\hline & 120 & & $0=0$ & C 260 & 000 & 0006 & 0896 \\
\hline & 160 & 0.880 & 0.500 & 0.508 & 0.880 & 0.886 & 0.886 \\
\hline & 200 & 0.886 & 0.506 & 0.368 & 0.886 & 0.886 & 0.886 \\
\hline
\end{tabular}

Table 14: Influence of failure rate $\left(\alpha_{6}\right)$ and repair rate $\left(\mu_{6}\right)$ of cement mill on system's profit with respect to time

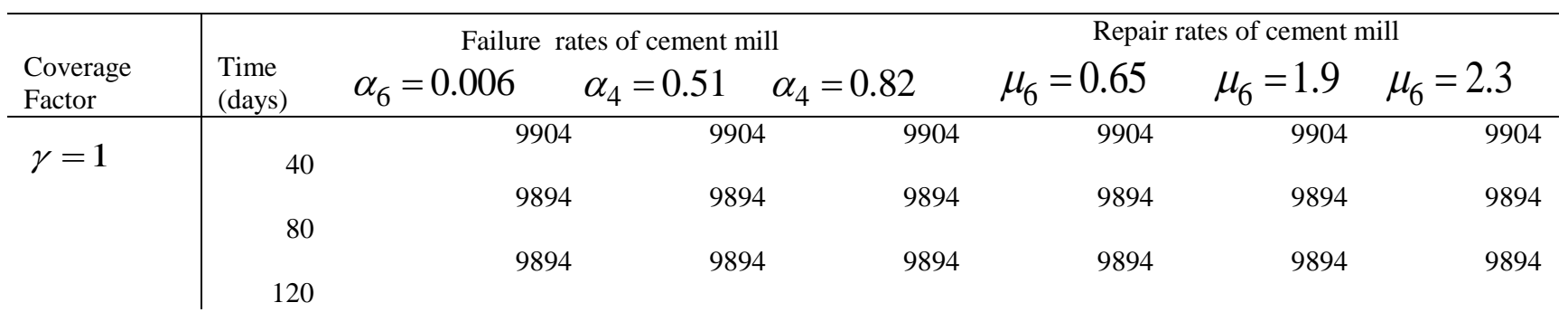




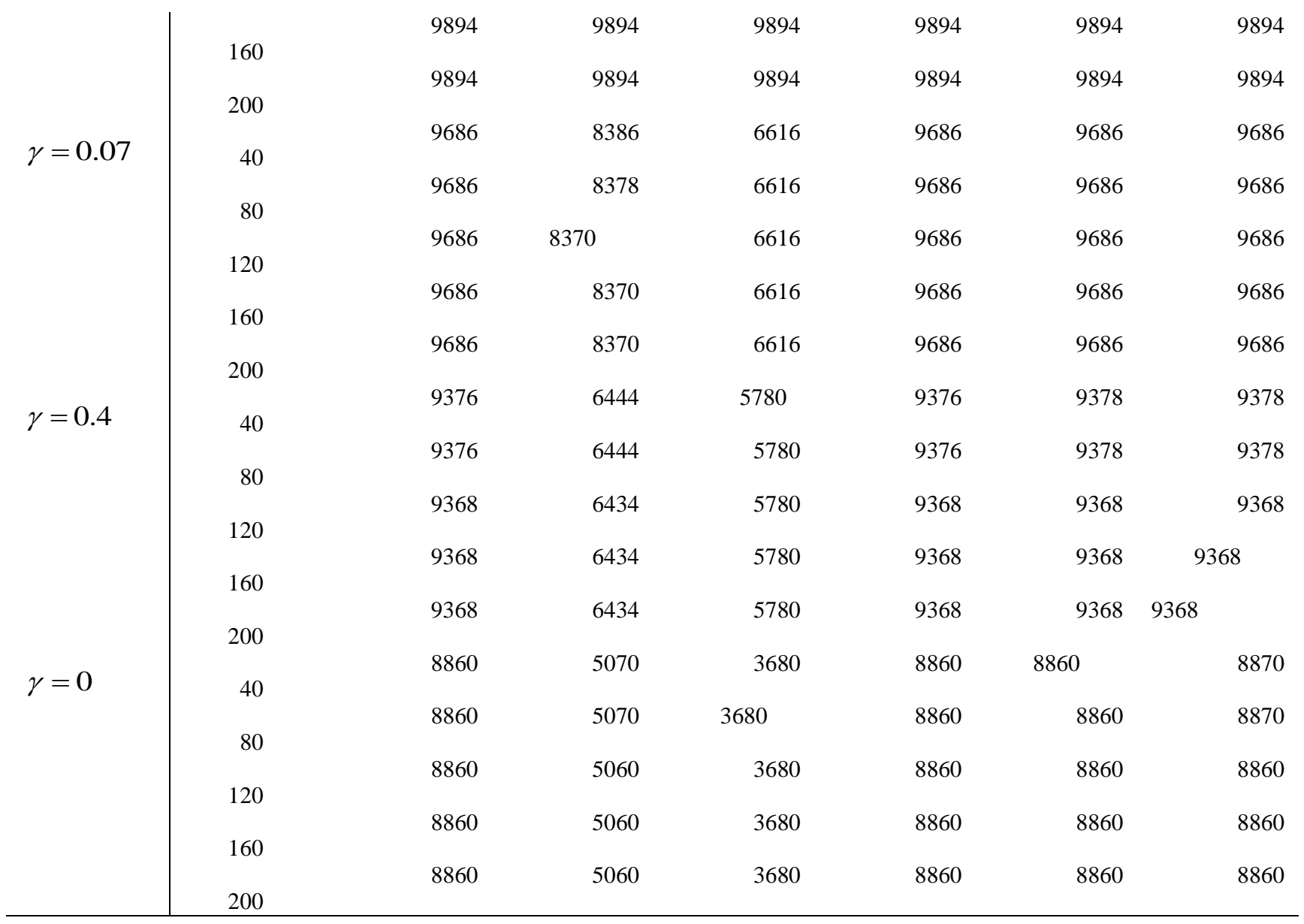

Influence of klin's partial failure and repair rate on availability and profit of cement manufacturing plant has been investigated for various values of failure and repair parameters. It is observed from table 15-16 that availability and profit increases with respect to increase of repair rate while decrease with respect to failure rates.

Table 15: Influence of klin's partial failure rate $\left(\alpha_{7}\right)$ and repair rate $\left(\mu_{7}\right)$ on system's availability with respect to time

\begin{tabular}{|c|c|c|c|c|c|c|c|}
\hline $\begin{array}{l}\text { Coverage } \\
\text { Factor }\end{array}$ & $\begin{array}{l}\text { Time } \\
\text { (days) }\end{array}$ & \multicolumn{2}{|l|}{ Failure rate of klin's partial } & & $\begin{array}{l}\text { Repair rates of } \\
\mu_{7}=0.9\end{array}$ & klin's partial $\mu$ & \\
\hline \multirow{5}{*}{$\gamma=1$} & 5 & 0.9904 & 0.9904 & 0.990 & 0.9904 & 0.990 & 0.990 \\
\hline & & 0.9894 & 0.9894 & 0.989 & 0.9894 & 0.989 & 0.989 \\
\hline & & 0.9894 & 0.9894 & 0.989 & 0.9894 & 0.989 & 0.989 \\
\hline & 120 & 0.9894 & 0.9894 & 0.98 & 0.9894 & 0.989 & 0.989 \\
\hline & & 0.9894 & 0.9894 & 0.98 & 0.9894 & 0.989 & 0.989 \\
\hline \multirow{3}{*}{$\gamma=0.07$} & 200 & 0.9686 & 0.8876 & 0.779 & 0.9686 & 0.971 & 0.975 \\
\hline & & 0.9686 & 0.8876 & 0.779 & 0.9686 & 0.971 & 0.974 \\
\hline & 120 & 0.9686 & 0.8876 & 0.779 & 0.9686 & 0.970 & 0.974 \\
\hline
\end{tabular}




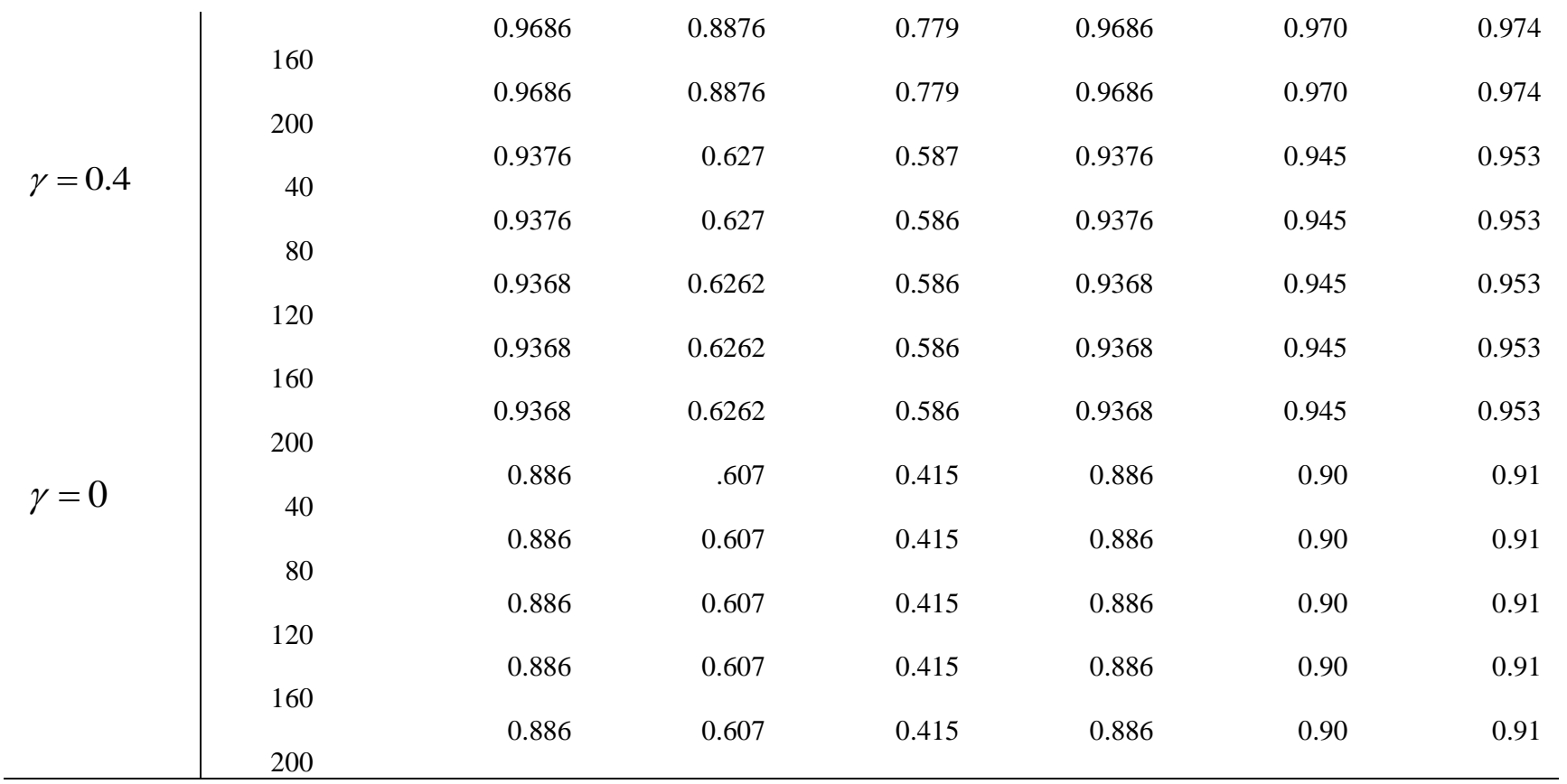

Table 16: Influence of klin's partial failure rate $\left(\alpha_{7}\right)$ and repair rate $\left(\mu_{7}\right)$ on system's profit with respect to time

\begin{tabular}{|c|c|c|c|c|c|c|c|}
\hline \multirow{2}{*}{$\begin{array}{l}\text { Coverage } \\
\text { Factor } \\
\gamma=1\end{array}$} & \multirow{2}{*}{$\begin{array}{r}\begin{array}{l}\text { Time } \\
\text { (days) }\end{array} \\
40\end{array}$} & \multirow{2}{*}{$\begin{array}{c}\text { Failure rate of klin's partial } \\
\alpha_{7}=0.004 \alpha_{7}=0.41 \\
9904\end{array}$} & \multicolumn{2}{|c|}{$\alpha_{7}=0.901$} & \multicolumn{3}{|c|}{$\begin{array}{l}\text { Repair rates of klin's partial } \mu_{7}=0.71 \\
\mu_{7}=0.9 \quad \mu_{7}=1.15\end{array}$} \\
\hline & & & 9904 & 9900 & 9904 & 9900 & 9900 \\
\hline & 80 & 9894 & 9894 & 9890 & 9894 & 9890 & 9890 \\
\hline & 120 & 9894 & 9894 & 9890 & 9894 & 9890 & 9890 \\
\hline & 160 & 9894 & 9894 & 9800 & 9894 & 9890 & 9890 \\
\hline & 200 & 9894 & 9894 & 9800 & 9894 & 9890 & 9890 \\
\hline \multirow[t]{5}{*}{$\gamma=0.07$} & 40 & 9686 & 8876 & 7790 & 9686 & 9710 & 9750 \\
\hline & 80 & 9686 & 8876 & 7790 & 9686 & 9710 & 9740 \\
\hline & 120 & 9686 & 8876 & 7790 & 9686 & 9700 & 9740 \\
\hline & 160 & 9686 & 8876 & 7790 & 9686 & 9700 & 9740 \\
\hline & 200 & 9686 & 8876 & 7790 & 9686 & 9700 & 9740 \\
\hline \multirow[t]{5}{*}{$\gamma=0.4$} & 40 & 9376 & 6270 & 5870 & 9376 & 9450 & 9530 \\
\hline & 80 & 9376 & 6270 & 5860 & 9376 & 9450 & 9530 \\
\hline & 120 & 9368 & 6262 & 5860 & 9368 & 9450 & 9530 \\
\hline & 160 & 9368 & 6262 & 5860 & 9368 & 9450 & 9530 \\
\hline & 200 & 9368 & 6262 & 5860 & 9368 & 9450 & 9530 \\
\hline \multirow[t]{5}{*}{$\gamma=0$} & 40 & 8860 & 6070 & 4150 & 8860 & 9000 & 9100 \\
\hline & 80 & 8860 & 6070 & 4150 & 8860 & 9000 & 9100 \\
\hline & 120 & 8860 & 6070 & 4150 & 8860 & 9000 & 9100 \\
\hline & 160 & 8860 & 6070 & 4150 & 8860 & 9000 & 9100 \\
\hline & 200 & 8860 & 6070 & 4150 & 8860 & 9000 & 9100 \\
\hline
\end{tabular}




\section{CONCLUSION}

Here, numerical results for availability and profit function of cement manufacturing plant has been analyzed. The availability and profit of cement plant helps system designers to improve the design for enhancing the reliability of plant. It is identified that coverage factor plays a prominent role in the enhancing the reliability of the plant. It is revealed that profit and availability increased with the increase of failure rates while it sharply declines with the increase of time and failure rates. Preheater, cooler, cement mill and kiln are the prominent subsystems that highly influence the availability and profit of the plant. So, it is recommended that by increasing repair rates, adopting proper maintenance strategies and arranging redundant unit plant can be made more available and profitable.

\section{REFERENCES}

[1] Saini M and Kumar A (2019) Performance analysis of evaporation system in sugar industry using RAMD analysis. J Braz Soc Mech Sci Eng 41:4

[2] Kumar, A. Goyal, D., Saini, M., Joshi. H. (2019). Reliability, maintainability and sensitivity analysis of physical processing unit of sewage treatment plant. SN Appl. Sci. 1: 1507. https://doi.org/10.1007/s42452019-1544-7

[3] Kumar A, Saini M (2018) Fuzzy availability analysis of a marine power plant. Materials Today: Proceedings. 5 (11P3): 25195-25202.

[4] Dahiya O, Kumar A, Saini M (2019) Mathematical modeling and performance evaluation of A-Pan crystallization system in a sugar industry. SN Applied Sciences, https://doi.org/10.1007/s42452-019-0348-0

[5] Gupta, N., Saini, M. \& Kumar, A. Operational availability analysis of generators in steam turbine power $\begin{array}{llll}\text { plants. SN } & \text { Appl. } & \text { Sci. 2, } 779 & \text { (2020). }\end{array}$ https://doi.org/10.1007/s42452-020-2520-y

[6] Goyal, D., Saini, M., Kumar, A. (2020). Reliability, Maintainability and Sensitivity Analysis of Biological and Chemical Processing Unit of Sewage Treatment Plant. International Journal of Advanced Science and
Technology, 29(3), 5399 - 5415.

[7] Saini M, Kumar A (2019) Performance analysis of evaporation system in sugar industry using RAMD analysis. J Braz Soc Mech Sci Eng 41:4

[8] Goyal, D., Kumar, A., Saini, M., Joshi. H. (2019). Reliability, maintainability and sensitivity analysis of physical processing unit of sewage treatment plant. SN Appl. Sci. 1: 1507. https://doi.org/10.1007/s42452019-1544-7

[9] Saini, M., Kumar, A. \& Shankar, V.G. A study of microprocessor systems using RAMD approach. Life Cycle Reliab Saf Eng 9, 181-194 (2020). https://doi.org/10.1007/s41872-020-00114-3

[10] Saini, M., Kumar, A. Stochastic Modeling of a SingleUnit System Operating Under Different Environmental Conditions Subject to Inspection and Degradation. Proc. Natl. Acad. Sci., India, Sect. A Phys. Sci. 90,319-326 (2020). https://doi.org/10.1007/s40010-018-0558-7

[11] Dahiya OM, Kumar AS, Saini MO. An analysis of feeding system of sugar plant subject to coverage factor. Int J Mech Prod Eng Res Dev (IJMPERD). 2019; 9 (1):495-508.

[12] Dahiya O, Kumar A, Saini M. Performance evaluation and availability analysis of a harvesting system using fuzzy reliability approach. Int J Recent Technol Eng. 2019;7(5):248-54

[13] GARG, Vikas; KADYAN, M S; KUMAR, Jitender. Stochastic Analysis of aTwo Non-Identical Units Redundant System with Preventive Maintenance and Priority. International Journal of Statistics and Reliability Engineering, [S.1.], v. 7, n. 1, p. 107-113, Jul. 2020.

[14] BARAK, M S.; YADAV, Dhiraj; BARAK, Sudesh K. Stochastic Analysis of a Cold Standby System with Conditional Failure of Server. International Journal of Statistics and Reliability Engineering, [S.1.], v. 4, n. 1, p. 65-69, Aug. 2017. 